\title{
A REVIEW OF A HUMAN FACTORS INTEGRATION APPORACH FOR THE INTRODUCTION OF COLLABORATIVE INDUSTRIAL ROBOTS IN MANUFACTURING SETTINGS
}

\author{
GEORGE CHARALAMBOUS and SARAH R. FLETCHER \\ Industrial Psychology and Human Factors Group, \\ Centre for Structures Assembly and Intelligent Automation \\ School of Aerospace, Transport and Manufacturing \\ Cranfield University \\ Bedford, UK, MK43 OAL \\ E-mail: george.ch@hotmail.co.uk; s.fletcher@cranfield.ac.uk \\ PHILIP WEBB \\ Centre for Structures Assembly and Intelligent Automation \\ School of Aerospace, Transport and Manufacturing \\ Cranfield University \\ Bedford, UK, MK43 OAL \\ E-mail:p.f.webb@cranfield.ac.uk
}

\begin{abstract}
The purpose of this paper is twofold: (a) to review the work carried out by the authors in identifying the key human factors that would enable the rollout of industrial collaborative robots in manufacturing settings at the organisational and the individual level; and, (b) to present the authors' work in linking the key organisational human factors affecting the successful introduction of industrial robots, with the three major factors affecting trust development between human users and industrial robots. The output provides HF practitioners a guiding framework to facilitate appropriate user trust calibration to the robotic teammate by taking into consideration that trust is a dynamic, and continuously evolving, construct which can be calibrated via training and user empowerment.
\end{abstract}

\section{Introduction: Industrial Human-Robot collaboration and the need for human factors integration}

Certain manufacturing processes require the abilities of the human worker to react to external influences, such as tolerances or process variations. However, humans lack accuracy, repeatability, speed and strength. Industrial robots, on the other hand, have the ability to handle high payloads, are very accurate, do not suffer from fatigue and can be used for repetitive and dangerous tasks. Thus in a collaborative industrial cell, human operators will perform the "value added work" while robots take over the "non-value added work" [1]. Combining the advantages of human workers and robots leads to the development of humanrobot collaboration (HRC). Successful implementation of industrial HRC can potentially increase production output, enhance quality and reduce product cost [3, 4]. However, close collaboration of human workers and industrial robots has traditionally been prevented largely due to safety concerns. Recent technological advancements have seen collaborative robots becoming more lightweight, compact and designed with human safety as a priority $[5,6]$ which will enable HRC potential. In light of these recent advances, health and safety regulations are also being advanced to reflect that in some circumstances it is viable to allow some closer cooperation between operators and industrial robots [2]. Thus, the landscape is changing and moving inevitably towards greater HRC implementations in future manufacturing systems. 
The traditional preventive health and safety default position to segregate robots from people in industry, means that the industry's understanding of how human operators are likely behave in more collaborative environments is still an area under development. The implementation of industrial HRC is arguably anticipated to be a radical manufacturing change when compared to the traditional view of industrial robotics. Such a step change should be expected to bring, not only technical challenges, but also from a human factors (HF) perspective. Merely introducing industrial robots with collaborative capabilities onto the shop floor will not ensure acceptance and effective use. These intelligent work systems will inevitably alter workers' job roles and organisational protocols. In this changing environment, HF practitioners have a crucial role to play. With the concept of industrial HRC still at its infancy, it is therefore crucial to understand the key human factors that need to be considered for the successful implementation of industrial HRC.

\section{A review of key human factors affecting the introduction of industrial robots}

Recent work by Charalambous, Fletcher and Webb (2017), [7] highlighted that the HF integration should focus at two levels: (i) the organisational level human factors, that is how organisations address human factors for rolling out technological changes; and (ii) the individual level factors, i.e. factors affecting human workers. The sections below provide a review of their findings.

\subsection{Organisational level human factors}

Evidence suggests that when new manufacturing technologies are introduced, the focus is disproportionally placed on the technical/engineering aspects, whilst appropriate and timely HF integration is often neglected. This unbalanced approach has resulted in a failure to grasp the full potential of the manufacturing technologies introduced $[8,9,10]$. Ironically, the problem does not appear to lie with the machine or the technology itself, but rather it is the inattention to the human element and the lack of a comprehensive HF integration strategy to forge its acceptance that appears to impede its success [11, 12, 13]. Charalambous (2014), [14] through a review of relevant literature from comparable domains captured the key organisational factors that would affect the successful rollout of industrial HRC in an initial theoretical framework shown below:

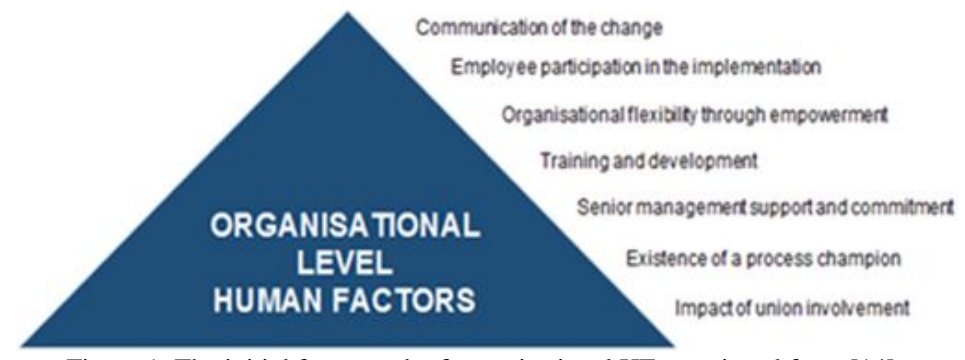

Figure 1. The initial framework of organisational HF - retrieved from [14].

Through an exploratory case study of comparable live industrial automation implementation in a high hazard industry, it was attempted to identify whether the organisational level human factors identified in the theoretical framework were either enablers or barriers in relation to implementation of HRC work. Furthermore, the case study aimed at identifying any additional human factors not captured in the theoretical framework. 


\subsubsection{Key findings}

Details of the study's findings were reported in [15] and a summary of the key findings is discussed in this section.

This work indicated that the organisational human factors illustrated in Figure 1 are applicable for the successful implementation of industrial HRC. For example, communication, employee participation and training provision were all found to be critical to the success of the rollout of industrial HRC.

At the same time additional, domain-specific, organisational human factors emerged along with inter-relation between them which are expanding on existing literature and are worthy of note, not least for industry HF practitioners:

1. Lack of awareness of the manual process complexity by the system integrator/supplier can be a major barrier towards providing a technically fit for purpose automated system. This is an important contribution of this research as this factor did not emerge in the literature review. The key point to note is that a system integrator/supplier may well possess expertise on the automated system they are supplying (e.g. industrial robot); however, they may not necessarily have the required expertise/knowledge of the domain in which that system is being deployed (e.g. manufacturing tasks requiring skilled workforce). In such occasions, an off-the-shelf automated system may not bridge the gap.

2. Following from the point above, the case study findings suggested that operator/user participation in the rollout is not only important as a means of enhancing acceptance of the new system amongst the workforce, but is vital to provide the system integrator with the vital knowledge of the manual processes (see point 1 above). Understanding how the operators perform the manual process thus identifying the key process variables in advance is a key step in order to successfully introduce a process capable automated system.

3. Senior management support and commitment was found to be linked with the allocation of the necessary resources for developing a technically fit for purpose automated system. This is an additional factor that did not appear in the theoretical framework. Resource allocation for assisting the system integrator with the development of the automated system is a critical step. This implies some compromises may need to be made, as these resources will not be contributing to the main production line. This is a challenging issue and difficult decisions will need to be made by the senior management team. Allocating the necessary resources for the development of the system is likely to impact production rates. The benefits of that, however, is that the system stands a better chance of reaching a process capable level sooner. Therefore, senior management will need to commit to the project and lead the way in terms of finding the common ground between the two.

4. Their case study findings appeared to link the importance of having a process champion with the communication of project information to key stakeholders. This was found to be an important step during the early stages when a higher level of uncertainty level is to be expected.

\subsection{Individual level human factors}

Similar issues, albeit of different nature, can be expected at the individual level. The literature review of human-automation and human-robot interaction provided a list of the key individual level factors which appear to be of most importance for the successful implementation of industrial HRC was summarised in [14] and is shown below: 


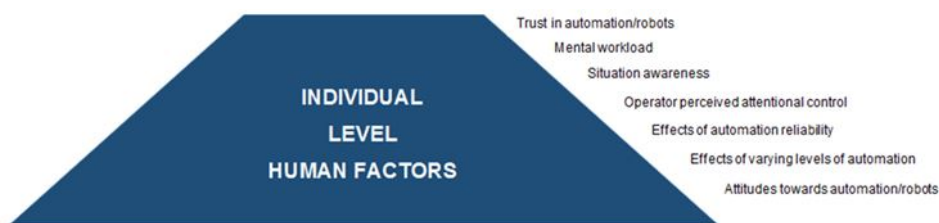

Figure 2. The individual level human factors - retrieved from [14].

Although each of these individual level human factors merit a standalone investigation, the construct of trust in automation/robots has been identified as being pivotal influencing the all the remaining [14]. For example, it has been demonstrated that if a robotic agent is perceived by the operator to be unreliable or unpredictable it will increase operator's mental workload $[16,17]$. In other words, if the operator does not have adequate trust in the robotic teammate, s/he is likely to place more mental resources on raw data to ensure the robot is taking the correct actions thus increasing mental workload. The same applies for situation awareness. In a human-robot collaboration scenario the robotic agent is envisaged to be responsible for a particular part of the task. Inherently, the human operator will have to rely that the robot is performing adequately. If for instance the operator does not trust the robot, s/he is likely to have to allocate additional attentional resources to monitor the robot thus potentially leading to performance degradation. Also, human attitudes towards robots represent a key element to enable their acceptance within our societies [18; 19]. Given that the construct of trust has been defined as the "attitude that an agent will help achieve an individual's goals in a situation characterized by uncertainty and vulnerability" [20], then implicitly trust becomes a central aspect towards building an appropriate attitude towards robotic teammates. Therefore, understanding how trust develops when individuals interact with industrial robots can be vital for the success of the collaboration.

In summary, the review suggested that trust would appear to be a central element among all of the identified factors. Although trust has been the topic of numerous studies over the years and was investigated in different contexts and possible antecedents of trust in human-robot interaction were identified [17], it had not yet been investigated whether these are relevant to the industrial context and what other context specific factors are crucial to consider. On this basis, Charalambous, Fletcher and Webb (2015) [21] developed a psychometric scale that measures trust specifically in industrial HRC. Such a measurement tool would not only allow us to quantify trust in industrial HRC, but it would also offer the opportunity to system designers to identify the key system aspects that can be manipulated to optimise trust in industrial HRC.

\subsubsection{Key findings: Trust development in industrial HRC}

The development of the psychometric trust scale was carried out in three phases. Initially, an exploratory study to collect participants' opinions when collaborating with industrial robots was carried out which led to the development of trust-related themes specifically related to industrial HRC. Based on these themes a pool of items was developed which were then placed on a rating survey. Then, a series of experimental case studies took place to quantify the key trust-related themes relevant to industrial HRC. The survey developed in the exploratory study was used to collect data. Finally, a quantitative analysis of the collected data was undertaken which led to the development of the trust scale. The developed psychometric scale indicated that trust in industrial HRC depends on three major factors:

- $\quad$ perceived safe cooperation

- $\quad$ perceived robot and gripping mechanism reliability 
- $\quad$ perceived robot's motion and pick up speed

The findings of this scale can have important practical implications. First, the tool offers the opportunity to quantify trust specifically in industrial HRC. Second, the three major factors identified in the scale highlight to system designers and engineers understand the key system characteristics that can affect operators' perception of trust in industrial HRC. For instance, the scale identified three key design aspects fostering trust industrial HRC namely, perceived safe cooperation, perceived robot and gripping mechanism reliability and perceived robot's motion. Therefore, particular emphasis needs to be given on these system characteristics. Third, this scale can assist to examine the relationship of each operator and enhance awareness regarding personal tendencies. For example, a poor score on a particular sub-scale (e.g. robot and gripping mechanism reliability) or on the entire scale can identify those operators in need for further training.

\section{Linking the organisational human factors to the trust scale findings}

At the organisational level, two of the key human factors that emerged were: (i) provision of training to the workforce and (ii) operator empowerment. These two factors can be utilised along with the developed trust scale to provide a tool with which operators' trust levels in the robotic teammate can be continuously calibrated. This is discussed in two parts:

- Part 1: discusses how the trust scale can be utilised in an initial training programme to assist operators' initial trust calibration.

- Part 2: discusses how operator empowerment is vital for continuous trust calibration which in turn will dynamically optimise operators' trust in the robotic teammate

\subsection{Part 1: Use the trust scale to the users' training programme}

In an industrial HRC scenario operators would be expected to share the same workspace and collaborate with an industrial robot to complete a task. An inaccurate or incomplete mental model can potentially lead the human operator to either overestimate or underestimate the abilities of the robotic teammate. This has been described in the literature as misuse (i.e. overestimation) and disuse (i.e. underestimation) [22). Both can be equally detrimental. The key is to achieve appropriate trust calibration. To calibrate appropriate trust in the robotic partner, it is vital for the human to hold a sufficiently developed mental model of the robot, whereby robot's capabilities are acknowledged [23). Therefore, to assist future human operators to develop a sufficient mental model of their robotic teammate, it is proposed to incorporate the trust scale findings in an operator training programme.

The aim of this training programme would be to provide operators with an understanding of the robot's abilities and limitations, rather than simply understanding how to use the robot to complete the process. This approach can help operators develop an appropriate mental model of the robot they will be requested to collaborate with. For instance, a key trust factor identified in the trust scale is the "perceived robot and gripping mechanism reliability". Does it mean that if the robot or the gripping mechanism is not $100 \%$ reliable all the time they are useless? Not quite. Automated systems, regardless of their safety integrity levels, may not always perform at $100 \%$ reliability due to technological limitations and/or due to software and hardware failures [24). Therefore, in a HRC scenario it should be expected that at some point, the robotic teammate's performance (i.e. the robot itself and/or the gripping mechanism) may be less than perfect. As Ososky and colleagues [23] have proposed, appropriate trust calibration is primarily influenced by the "human's mental model of the robot's ability and limitations, than the ground-truth reliability of the robot itself” (p.63). In other words, 
perception and reality are not necessarily the same trust can be heavily driven by user's perception of the robot irrespective of whether this perception is correct, partially correct or completely incorrect [25].

Therefore, an initial training programme, before the implementation of the robotic system, could be used as a strategy to raise operators' awareness regarding the ability and limitations of the robot and assist matching operators' perceptions with the system's actual capabilities. It has been indicated that optimal trust levels can be achieved when the users' perceptions of machine characteristics reflect the actual machine characteristics [26]. Lack of this knowledge will leave the operator with an incomplete mental model which in turn will make the robot's actions (or inactions) unpredictable thus significantly reducing trust in the robotic partner.

\subsection{Part 2: Operator empowerment to dynamically calibrate trust}

The development of mental models is a dynamic process and these models are refined through continuous interaction [23]. Trust, however, is a dynamic construct and evolves over time from dispositional (i.e. upon first encounter) to history-based trust (i.e. cumulative collaboration) [25]. As this transition occurs, users retrieve history-based mental models to interpret the actions of the system they are working with. Therefore, if the mental models created during the subsequent exposure (i.e. history-based) are not sufficiently developed, this is likely to lead to trust miscalibration. In an industrial HRC scenario, the more operators are collaborating with a robot, the more likely it is to experience a variety of real failure, errors or system deviation scenarios (particularly during the early stages of implementation). While these events occur, it is vital for operators to understand the sources of these events and the possible outcome of these events (whether a failure, error, or deviation). Also, through exposure they will be in a position to identify factors that diminish or enhance the robot's ability to perform as well as detect cues that suggest a potential malfunction. It has been recommended that trust can be calibrated by providing an accurate understanding of the factors that may lead the robot to fail and the outcomes of those failures [27]. To leverage this potential and enable effective HRC, it is proposed that operator empowerment can be a key strategy.

Operator empowerment was found to be one of the key enabling organisational human factors in the exploratory case study. Literature has suggested that, in a highly complex system, higher operator control and empowerment once the system is implemented will lead to operators obtaining a better understanding of the new system and task requirement [28). Through operator empowerment, the operators' already established mental model of the robot (from the initial training programme) will be updated based on their history of collaboration. If on the other hand, operators are not empowered, then they are likely to be alienated from the system which arguably may well turn them into passive "button-pushers" as opposed to active/empowered users of the system. This is likely to reduce system acceptance, but can also reduce their ability to develop an in-depth understanding of the system's source of events (i.e. failures, errors, deviations). Subsequently, their ability to recalibrate their trust is reduced leaving them with an incomplete mental model.

Finally, the knowledge gained by the operators, can then be passed into the training programme. In this approach the training programme of future novice operators is updated with real event scenarios, thus accelerating appropriate trust calibration of novice operators during the initial training programme by enabling greater match between their perceptions of the system and the actual system's capabilities. 


\section{Conclusions}

The purpose of this paper is twofold: (a) to review the work carried out by the authors identifying the key human factors that would enable the rollout of industrial collaborative robots in manufacturing settings at the organisational and the individual level; and, (b) to document the work carried out by the authors in linking the organisational human factors with trust in robots - a central theme of the individual level human factors.

The output of this work provides HF practitioners with the following:

- A review of the key organisational HF that were identified by the authors in an exploratory case study as being important to consider during the roll out of industrial HRC in manufacturing settings;

- A review of the psychometric scale developed by the authors to evaluate trust in the specific context of industrial HRC - this informs practitioners on the key design features of an industrial robot that have been found to influence human trust;

- A guiding framework which links these findings together to assist appropriate operator trust calibration by taking into consideration the notion that trust is a dynamic, and continuously evolving, construct which can be calibrated via training and user empowerment.

\section{Acknowledgments}

This research was funded by the EPSRC Centre for Innovative Manufacturing in Intelligent Automation. Special thanks to the industrial sponsors of the project for assisting with the data collection.

George Charalambous is currently a Senior Human Factors consultant at SNC-Lavalin Rail \& Transit, London, UK.

\section{References}

1 Unhelkar VV, Perez J, Boerkoel JC, Bix J, Bartscher S, Shah JA (2014) Towards control and sensing for an autonomous mobile robotic assistant navigating assembly lines International Conference on Robotics and Automation (ICRA).

2 International Organisation for Standardisation (2011) Robots and robotic devices - Safety requirements for industrial robots, Part 1: Robots. Geneva, Switzerland: International Standards Organisation.

3 Papadopoulos AV, Bascetta L, Ferretti G (2013) Generation of Human Walking Paths. International Conference on Intelligent Robots and Systems (IROS), Tokyo, Japan.

$4 \quad$ Weidner R, Kong N, Wulfsberg JP (2013) Human Hybrid Robot: a new concept for supporting manual assembly tasks. Production Engineering Research Development, 7(6):675-684.

5 Bostelman R, Shackleford W (2010) Improved performance of an automated guided vehicle by using a smart diagnostics tool. International Conference on Industrial Technology (ICIT), Vi a del Mar, Chile.

6 Guizzo E (2008) Three engineers, hunders of robots, one warehouse. IEEE Spectrum, 45(7):26-34.

7 Charalambous, G., Fletcher, S. R., \& Webb, P. (2017). The development of a Human Factors Readiness Level tool for implementing industrial human-robot collaboration. The International Journal of Advanced Manufacturing Technology, 91(5-8), 2465-2475.

8 Zammuto RF, O’Connor EJ (1992) Gaining advanced manufacturing technologies’ benefits: the roles of organization design and culture. Academy of Management Review, 17(4):701-728.

9 Chung CA (1996) Human issues influencing the successful implementation of advanced manufacturing technology. Journal of Engineering and Technology Management, 13(3):283-299.

10 McDermott CM, Stock GN (1999) Organisational culture and advanced manufacturing technology implementation. Journal of Operations Management, 17:521-533. 
Ghani KA, Jayabalan V (2000) Advanced manufacturing technology and planned organisational change. The Journal of High Technology and Management Research, 11(1): 1-18. Waldeck NE (2000) Advanced manufacturing technologies and workforce development. Garland Press, New York.

13 Lewis MW, Boyer KK (2002) Factors impacting AMT implementation: An integrative and controlled study. Journal of Engineering and Technology Management, 19:111-130 Charalambous, G (2014): The development of a human factors tool for the successful implementation of industrial human-robot collaboration. PhD Thesis. Cranfield University. Charalambous, G., Fletcher, S., \& Webb, P. (2015). Identifying the key organisational human factors for introducing human-robot collaboration in industry: an exploratory study. The International Journal of Advanced Manufacturing Technology, 81(9-12), 2143-2155. Miller, C. A., \& Parasuraman, R. (2007). Designing for flexible interaction between humans and automation: Delegation interfaces for supervisory control. Human Factors: The Journal of the Human Factors and Ergonomics Society, 49(1), 57-75

Hancock, P. A., Billings, D. R., Oleson, K. E., Chen, J. Y., De Visser, E., \& Parasuraman, R. (2011). A meta-analysis of factors influencing the development of human-robot trust. Aberdeen Proving Ground, MD 21005-5425: U.S. Army Research Laboratory Nomura, T., Kanda, T., \& Suzuki, T. (2006). Experimental investigation into influence of negative attitudes toward robots on human-robot interaction. AI \& Society, 20(2), 138-150. Fink, J., Bauwens, V., Kaplan, F., \& Dillenbourg, P. (2013). Living with a vacuum cleaning robot: A 6month ethnographic study. International Journal of Social Robotics, 5(3), 389-408 Lee, J. D., \& See, K. A. (2004). Trust in automation: Designing for appropriate reliance. Human Factors: The Journal of the Human Factors and Ergonomics Society, 46(1), 50-80. Charalambous, G., Fletcher, S., \& Webb, P. (2016). The development of a scale to evaluate trust in industrial human-robot collaboration. International Journal of Social Robotics, 8(2), 193-209. Parasuraman, R., \& Riley, V. (1997). Humans and automation: Use, misuse, disuse, abuse. Human Factors: The Journal of the Human Factors and Ergonomics Society, 39(2), 230-253 Ososky, S., Schuster, D., Phillips, E., \& Jentsch, F. (2013). Building appropriate trust in human-robot teams. Trust and Autonomous Systems AAAI Spring Symposium (pp. 60-65). AAAI Wickens, C. D., Huiyang, L., Santamaria, A., Sebok, A., \& Sarter, N. (2010). Stages and levels of automation: An integrated meta-analysis. In the Proceedings of the 54th Annual Human Factors and Ergonomics Society Meeting, (pp. 383-393) Merritt, S. M., \& Ilgen, D. R. (2008). Not all trust is created equal: Dispositional and history-based trust in human-automation interactions. Human Factors: The Journal of the Human Factors and Ergonomics Society, 50(2), 194-210 Madhavan, P., \& Wiegmann, D. A. (2007). Similarities and differences between human-human and human-automation trust: An integrative review. Theoretical Issues in Ergonomics Science, 8(4), 277-301 Ososky, S., Sanders, T., Jentsch, F., Hancock, P., \& Chen, J. Y. (2014). Determinants of system transparency and its influence on trust in and reliance on unmanned robotic systems. Proceedings of SPIE, Unmanned Systems Technology XVI, 90840E, (pp. 1-12) uncertainty: A theoretical integration. Applied Psychology: An International Review, 51(1), 146-169 